\title{
REFINED QUANTUM INVARIANTS FOR THREE-MANIFOLDS WITH STRUCTURE
}

\author{
CHRISTIAN BLANCHET \\ Département de Mathématiques \\ 2 rue de la Houssinière \\ BP 92208 \\ F-44322 Nantes Cedex 3, France \\ E-mail: blanchet@math.univ-nantes.fr
}

Introduction. Following Witten's interpretation ([Wi]) of the Jones polynomial ([Jo]) in terms of Topological Quantum Field Theory, Reshetikhin and Turaev ([RT]) and then many others have constructed invariants of 3-manifolds now called Quantum Invariants (see [Tu2] for a detailed exposition, and [Vo] for a survey). The construction of Reshetikhin and Turaev involves representation theory of quantum groups. This point of view gives a deep insight into the algebraic questions related to the subject, however it is not immediately accessible for the beginner. Among these quantum invariants those called the $S U(2)$-invariants can be obtained easily from the skein theory associated with the Kauffman bracket ([Ka]). This was first observed by Lickorish ([Li1],[Li2],[Li3]) and then systematically studied in [BHMV1]. Section 1 deals with this skein method. Starting with a formal skein theory, we discuss the construction of 3-manifolds invariants, and give the simplest examples. We think that this could be helpful for the beginner and hope that the method will be applied to new examples.

Once one has constructed a lot of 3-manifold invariants, the question is to understand their meaning, and this is far from clear at the moment. Let us discuss the example of $\tau^{S U(2)}$ at $q=e^{\frac{i \pi}{8}}([\mathrm{KM}])$ which corresponds to $\theta_{8}$ in [BHMV1] and [Bl1]. This invariant decomposes as a sum, over all spin structures on the manifold, of spin invariants. Moreover the spin invariant is (a version of) the well known Rochlin invariant. This was first observed by Kirby and Melvin and generalized independently in [KM], [Tu1] and [Bl1]. This example shows that considering refined invariants can help in understanding their geometrical meaning. Section 2 is about cohomological refinements of quantum invariants. According to H. Murakami $([\mathrm{Mu}]) \tau_{r}^{S U(n)}$ admits such refinements, for conveniently chosen

1991 Mathematics Subject Classification: Primary 57N10; Secondary 57N25.

The paper is in final form and no version of it will be published elsewhere. 
$r$. He states also a decomposition formula in which cohomology classes are replaced by some spin type structures. In section 3, a topological definition of these structures is given. In spite of its simplicity, this description seems to be new.

In this paper we only consider invariants of 3-manifolds. We will say in section 4 a few words about extending this to a whole Topological Quantum Field Theory. Following the methods developed in [BHMV3] together with N. Habegger, G. Masbaum and P. Vogel, we have, in a joint work with G. Masbaum ([BM]), constructed and studied this extension, for the spin refined invariants obtained from the Kauffman bracket. Understanding other refined theories is our challenge!

The content of this paper was exposed at the Mini-semester in Knot Theory in Warsaw (Summer 1995). We thank the organizers and the Stefan Banach Center for their invitation and hospitality.

1. Three-manifolds invariants derived from a skein theory. Various skein modules of 3-manifolds have been defined and studied (see $[\mathrm{P}],[\mathrm{HP}]$ ). In each case the modules have presentations in which generators are links, and relations are local (skein) relations between them. The definition below gives a general nonsense. We emphasize the functorial property. Here we consider embeddings of 3-manifolds. In a concrete theory generated by links, an embedding $M \rightarrow M^{\prime}$ carries links in $M$ to links in $M^{\prime}$.

Let $\mathcal{S}$ be a functor from the category of compact oriented 3-manifolds with isotopy classes of oriented embeddings to the category of $k$-modules. We will say that $\mathcal{S}$ is a skein theory if $\mathcal{S}$ is monoidal and involutive (see [ML]). Here $k$ is a commutative ring with unit, supposed to be equipped with an involutive automorphism $\lambda \mapsto \bar{\lambda}$ ).

Remark 1. The monoidal property says that, up to canonical isomorphisms, one has $\mathcal{S}\left(M_{1} \amalg M_{2}\right)=\mathcal{S}\left(M_{1}\right) \otimes \mathcal{S}\left(M_{2}\right)$ and $\mathcal{S}(\emptyset)=k$. Using the embedding $\varepsilon: \emptyset \rightarrow M$, we get the vector $\mathcal{S}(\varepsilon)(1) \in \mathcal{S}(M)$. In a concrete skein theory, defined using links, this vector is represented by the empty link in $M$. We will denote it by $\emptyset$.

Remark 2. Involutivity says that, up to a canonical isomorphism, the module $\mathcal{S}(-M)$ is equal to $\overline{\mathcal{S}(M)}$. If a fixed oriented diffeomorphism $g: M \stackrel{\sim}{\rightarrow}-M$ is given, then $\mathcal{S}(g)$ defines an anti-linear automorphism of $\mathcal{S}(M)$ (a linear isomorphism from $\mathcal{S}(M)$ to $\overline{\mathcal{S}(M)})$. This automorphism will be called the mirror and denoted $x \mapsto \bar{x}$. The map $(z, \alpha) \mapsto(\bar{z}, \alpha)$ gives such a $g$, in $\mathbf{D}^{2} \times \mathbf{S}^{1}$ as well as in $\mathbf{S}^{3} \subset \mathbf{C}^{2}$.

Remark 3 . Using any oriented embedding $\mathbf{D}^{3} \amalg \mathbf{D}^{3} \rightarrow \mathbf{D}^{3}$ we get a product on the module associated with the 3 -ball $\mathcal{S}\left(\mathbf{D}^{3}\right)$. We also get a product on the module $\mathcal{S}\left(\mathbf{D}^{2} \times \mathbf{S}^{1}\right)$, by using a standard embedding $\mathbf{D}^{2} \times \mathbf{S}^{1} \amalg \mathbf{D}^{2} \times \mathbf{S}^{1} \rightarrow \mathbf{D}^{2} \times \mathbf{S}^{1}$. This makes $\mathcal{S}\left(\mathbf{D}^{3}\right)$ and $\mathcal{S}\left(\mathbf{D}^{2} \times \mathbf{S}^{1}\right)$ into commutative algebras with unit $1=\emptyset$.

To an oriented embedding of a disjoint union of solid tori

$$
g=\coprod_{i=1}^{m} g_{i}: \coprod_{i=1}^{m} \mathbf{D}_{i}^{2} \times \mathbf{S}_{i}^{1} \rightarrow M
$$

is associated a multilinear map

$$
\mathcal{S}(g): \mathcal{S}\left(\mathbf{D}^{2} \times \mathbf{S}^{1}\right)^{\otimes m} \rightarrow \mathcal{S}(M)
$$


By the isotopy hypothesis, this map only depends on the framed link $L=\left(L_{1}, \ldots, L_{m}\right)$ underlying $g$.

We call a bracket any linear map $\langle\ldots\rangle: \mathcal{S}\left(\mathbf{S}^{3}\right) \rightarrow k$ involutive and such that the composition

$$
\mathcal{S}\left(\mathbf{D}^{3}\right) \rightarrow \mathcal{S}\left(\mathbf{S}^{3}\right) \rightarrow k
$$

is multiplicative. Here involutive means that the mirror image (see remark 2 above) is sent to the conjugate.

Notations. As already explained, a framed link $L=\left(L_{1}, \ldots, L_{m}\right)$ in the sphere gives a multilinear map $\mathcal{S}\left(\mathbf{D}^{2} \times \mathbf{S}^{1}\right)^{\otimes m} \rightarrow k$. The image of $x_{1} \otimes \ldots \otimes x_{m}$ by this map is denoted by $\left\langle L_{1}\left(x_{1}\right), \ldots, L_{m}\left(x_{m}\right)\right\rangle$ or $\left\langle L\left(x_{1}, \ldots, x_{m}\right)\right\rangle$. Such an element is said to be obtained by skein cabling, or simply by cabling.

For $\epsilon \in\{-1,0,1\}$ we note $U_{\epsilon}$ the unknot with framing $\epsilon$, and $H_{\epsilon}$ the Hopf link with linking number one and both components having framing $\epsilon$.

A framed link $L$ determines by surgery a 3-manifold which will be denoted by $S^{3}(L)$ (every compact oriented 3-manifold can be obtained in this way). As a consequence of Kirby's theorem ([Ki]), we have the following proposition.

Proposition 1.1. If $\omega \in \mathcal{S}\left(\mathbf{D}^{2} \times \mathbf{S}^{1}\right)$ satisfies

(K) $\forall x \in \mathcal{S}\left(\mathbf{D}^{2} \times \mathbf{S}^{1}\right)\left\langle H_{1}(x, \omega)\right\rangle=\left\langle U_{0}(x)\right\rangle\left\langle U_{1}(\omega)\right\rangle$ and $\left\langle U_{1}(\omega)\right\rangle$ is invertible then

$$
\frac{\langle L(\omega, \ldots, \omega)\rangle}{\left\langle U_{1}(\omega)\right\rangle^{b_{+}}\left\langle U_{-1}(\omega)\right\rangle^{b_{-}}}
$$

is an invariant of the surgered manifold $M=\mathbf{S}^{3}(L)$. Here $b_{+}\left(\right.$resp. $\left.b_{-}\right)$is the number of positive (resp. negative) eigenvalues of the linking matrix $B_{L}$ associated with $L$.

Remark. The vector $\omega$ is defined up to a multiplicative invertible factor $\lambda$ and up to the kernel $\mathcal{N}_{1}$ of the bilinear form $\left\langle H_{1}(),\right\rangle$. The factor $\lambda$ multiplies the invariant by $\lambda^{b_{1}(M)}$ where $b_{1}(M)$ is the first Betti number of $M$. Adding an element of $\mathcal{N}_{1}$ does not change the invariant; this is a corollary of the following lemma whose proof will be given forward.

LEMma 1.2. If $(K)$ has a solution, then for any framed link in $\mathbf{S}^{3}$ the multilinear form $\langle L(\ldots)\rangle$ is well defined on the quotient $\mathcal{S}\left(\mathbf{D}^{2} \times \mathbf{S}^{1}\right) / \mathcal{N}_{1}$.

Remark. Understanding the kernel $\mathcal{N}_{1}$ is a key point in this construction. Multiplying by any $x$, in the algebra $\mathcal{S}\left(\mathbf{D}^{2} \times \mathbf{S}^{1}\right)$, is a self-adjoint operator with respect to $\left\langle H_{1}(),\right\rangle$, thus $\mathcal{N}_{1}$ is an ideal. The first condition in $(\mathrm{K})$ can be written

$$
\forall x\left(x-\left\langle U_{0}(x)\right\rangle\right) \omega \in \mathcal{N}_{1}
$$

Moreover it is sufficient above to consider $x$ in a set of generators of $\mathcal{S}\left(\mathbf{D}^{2} \times \mathbf{S}^{1}\right)$ as an algebra.

About the proof of proposition 1.1. This proposition can be proved using the well known Kirby theorem [Ki] as refined in [FR] and [RT] (see [Tu2] ch. 2). There are two non standard points here. 
First one must check that changing the orientation of one component of the link $L$ does not modify the value of $\left\langle L_{1}(\omega), \ldots, L_{m}(\omega)\right\rangle$. This will follows from lemma 1.3 .

Second one must show that $(\mathrm{K})$ implies that $\left\langle U_{-1}(\omega)\right\rangle$ is also invertible. This is a consequence of lemma 1.4 .

LEMMA 1.3. If $\omega$ is a solution of $(K)$, then the skein element $\check{\omega}=\mathcal{S}(j)(\omega)$, where $j$ is the diffeomorphism $(z, \alpha) \mapsto(\bar{z}, \bar{\alpha})$ of $\mathbf{D}^{2} \times \mathbf{S}^{1}$, is equal to $\omega$ in the quotient $\mathcal{S}\left(\mathbf{D}^{2} \times \mathbf{S}^{1}\right) / \mathcal{N}_{1}$.

LEMMA 1.4. If $\omega$ is a solution of $(K)$. Let $\bar{\omega} \in \mathcal{S}\left(\mathbf{D}^{2} \times \mathbf{S}^{1}\right)$ be the mirror image of $\omega$. In the quotient $\mathcal{S}\left(\mathbf{D}^{2} \times \mathbf{S}^{1}\right) / \mathcal{N}_{1}$, one has $\bar{\omega}=\lambda \omega$ with $\lambda \bar{\lambda}=1$.

Recall (see remark 2 above) that $\bar{\omega}$ is defined using the diffeomorphism $(z, \alpha) \mapsto(\bar{z}, \alpha)$.

As a consequence, after multiplying by a square root of $\lambda$ (extend the scalars if necessary), we get a solution of $(\mathrm{K})$ equal to its mirror image. The corresponding invariant of 3-manifolds is then involutive ( $M$ and $-M$ have conjugate invariants).

Proof of lemma 1.3. Let us denote, for $x \in \mathcal{S}\left(\mathbf{D}^{2} \times \mathbf{S}^{1}\right), \mathcal{S}(j)(x)=\check{x}$. By isotopy, for any $x \in \mathcal{S}\left(\mathbf{D}^{2} \times \mathbf{S}^{1}\right)$,

$$
\left\langle U_{\epsilon}(\check{x})\right\rangle=\left\langle U_{\epsilon}(x)\right\rangle \quad \epsilon \in\{0,1\} \text {, and }\left\langle H_{1}(x, \check{\omega})\right\rangle=\left\langle H_{1}(\check{x}, \omega)\right\rangle
$$

It follows that $\check{\omega}$ is a solution of $(\mathrm{K})$, equal to $\omega$ up to $\mathcal{N}_{1}$.

Notations. A right handed twist induces an automorphism of $\mathcal{S}\left(\mathbf{D}^{2} \times \mathbf{S}^{1}\right)$ denoted by $t$. For $x \in \mathcal{S}\left(\mathbf{D}^{2} \times \mathbf{S}^{1}\right)$, let $c_{x}$ be the operator defined by $c_{x}(y)=h(x \otimes y)$, where $h$ is induced by a link in the torus $\mathbf{D}^{2} \times \mathbf{S}^{1}$ whose first component is parallel to a meridian and whose second component is the core of the torus (standardly framed). Note that $t$ and $c_{x}$ commute.

The map $c=\left[x \mapsto c_{x}\right]$ is a representation of $\mathcal{S}\left(\mathbf{D}^{2} \times \mathbf{S}^{1}\right)$ onto itself, dual to multiplication with respect to the bilinear form $\left\langle H_{0}(),\right\rangle$. Namely

$$
\forall x, y, z \in \mathcal{S}\left(\mathbf{D}^{2} \times \mathbf{S}^{1}\right)\left\langle H_{0}\left(c_{x}(y), z\right)\right\rangle=\left\langle H_{0}(y, x z)\right\rangle
$$

By computing in two different ways the expression $\left\langle H_{0}(t(x) t(\check{\omega}), t(\omega))\right\rangle$ we have.

LEMMA 1.5. If $\omega$ is a solution of $(\mathrm{K})$, then

$$
\forall x \in \mathcal{S}\left(\mathbf{D}^{2} \times \mathbf{S}^{1}\right) \quad\left\langle H_{0}(x, \omega)\right\rangle=\left\langle H_{0}(t(x), \omega)\right\rangle
$$

Proof of lemma 1.4. Using the lemma above, we have first

$$
\left\langle H_{0}(x \omega, \omega)\right\rangle=\left\langle H_{0}(t(x \omega), \omega)\right\rangle=\left\langle U_{0}(x)\right\rangle\left\langle U_{-1}(\omega)\right\rangle\left\langle U_{1}(\omega)\right\rangle
$$

We have also

$$
\left\langle H_{0}(x \omega, \omega)\right\rangle=\left\langle H_{0}\left(\omega, c_{x}(\omega)\right)\right\rangle=\left\langle H_{0}\left(\omega, t c_{x}(\omega)\right)\right\rangle=\left\langle H_{0}(x \omega, t(\omega))\right\rangle=\left\langle U_{-1}(x \omega)\right\rangle\left\langle U_{1}(\omega)\right\rangle
$$

Hence

$$
\forall x \in \mathcal{S}\left(\mathbf{D}^{2} \times \mathbf{S}^{1}\right) \quad\left\langle U_{-1}(\bar{x} \omega)\right\rangle=\left\langle U_{0}(\bar{x})\right\rangle\left\langle U_{-1}(\omega)\right\rangle
$$

Using the mirror automorphism we show that $\bar{\omega}$ is a solution of $(\mathrm{K})$. Thus, modulo $\mathcal{N}_{1}$, $\bar{\omega}=\lambda \omega$. Taking the mirror once more, $\omega=\lambda \bar{\lambda} \omega$. Using invertibility of $\left\langle U_{1}(\omega)\right\rangle$, we have $\lambda \bar{\lambda}=1$. 
Note. The drawings corresponding to the computation above prove the refinement of Kirby's theorem ([RT]) saying that negative Fenn-Rourke moves can be deduced from positive and special negative ones.

Proof of lemma 1.2. Let $L=\left(L_{1}, \ldots, L_{m}\right)$ be a framed link in $\mathbf{S}^{3}$, we have to show that $\left\langle L_{1}\left(x_{1}\right), \ldots, L_{m}\left(x_{m}\right)\right\rangle$ is zero if $x_{1}$ is in $\mathcal{N}_{1}$. The proof is in three steps.

If $L_{1}=U_{1}$, the definition of $\mathcal{N}_{1}$ gives the result.

Then using the properties of $\omega$ it is shown that $\mathcal{N}_{1}$ is fixed by the automorphism $t$ of $\mathcal{S}\left(\mathbf{D}^{2} \times \mathbf{S}^{1}\right)$ induced by a right handed twist. This gives the result if $L_{1}$ is the unknot with any framing.

In the general case, the component $L_{1}$ can be unknotted by changing some crossings. Inserting an $\omega$ around each changed crossing reduces the problem to the preceding case.

EXAMPLE 1. Kauffman bracket skein theory. Given an invertible element $A$ in a ring $k$ (equipped with an involution sending $A$ to $A^{-1}$ ), the skein module $\mathcal{K}(M)$ is the free $k$ module generated by isotopy classes of banded links (embedded copies of $\mathbf{S}^{1} \times[0,1]$ ), quotiented by the usual Kauffman relations ([Ka]). The equation $(\mathrm{K})$ has been discussed in [BHMV1]. The result is that $A$ must be a root of unity whose order is an even integer $2 p$. For each $p$ there is, up to changing the ring and normalizing, a unique invariant $\theta_{p}$. In the notation coming from Chern-Simons gauge theory, $\theta_{p}$ corresponds to the $S U(2)$-invariant for even $p$ and to the $S O(3)$-invariant for odd $p$.

EXAMPLE 2. Skein theory associated with linking. Let $q$ be an invertible element in $k$ (equipped with an involution sending $q$ to $q^{-1}$ ). Define the skein module $\mathcal{L}(M)$ to be the free $k$-module generated by isotopy classes of framed links, quotiented by the local relations

$$
L_{+}=q L_{0} \quad L_{-}=q^{-1} L_{0} \quad L \amalg U_{0}=L
$$

Here $L_{+}, L_{-}$et $L_{0}$ are the same except in a ball $D^{2} \times[0,1]$ where their projection on the disc $D^{2} \times\{0\}$ have respectively, a positive crossing, a negative crossing and no crossing; $U_{0}$ is an unknot with the framing given by the disc it bounds. The algebra $\mathcal{L}\left(\mathbf{D}^{2} \times \mathbf{S}^{1}\right)$ is isomorphic to $k\left[y, y^{-1}\right]$. The condition $(\mathrm{K})$ has a solution only if $q$ is a root of unity whose order is either an odd integer $N$, or is $2 N$ with $N$ even. In each case, if $N$ is invertible in $k$, an invariant is produced, which is equal to the $Z_{N}$-invariant derived from linking matrices in $[\mathrm{MOO}]$ (see also $[\mathrm{MPR}]$ ).

EXAMPLE 3. HOMFLY theory. Quantum $S U(n)$-invariants of 3-manifolds have been obtained by Turaev and Wenzl ([TW]) and studied by Kohno and Takata $([\mathrm{KT}])$. Recently, following a combinatorial approach of Morton ([Mo]), Yokota ([Yo]) gave a construction of this invariants based on HOMFLY skein theory. The case $n=3$ was already given by Ohtsuki and Yamada $([\mathrm{OY}])$. The construction of Yokota enters easily in our description.

The $S U(n)$ specialized HOMFLY skein module of a 3-manifold $M$ is defined to be the free module generated by framed links in $M$, quotiented by the relations

$$
a L_{+}-a^{-1} L_{-}=\left(a^{n}-a^{-n}\right) L_{0}
$$




$$
\begin{aligned}
L \amalg U_{0} & =\frac{a^{n^{2}}-a^{-n^{2}}}{a^{n}-a^{-n}} L \\
L^{+f} & =a^{f\left(n^{2}-1\right)} L
\end{aligned}
$$

In the first two relations, notations are standards; in the third one $L^{+f}$ is obtained from $L$ by adding the integer $f$ to the framing. Here $a$ is an invertible element in $k$ (equipped with an involution sending $a$ to $a^{-1}$ ).

If $a$ is a primitive $(k+n) n$-th root of unity, then Lemma 3.2, and Proposition 4.3 in $[\mathrm{Yo}]$ show that $(\mathrm{K})$ has a solution. The needed computation is related with combinatorics in the algebra of Young diagrams studied by Morton and Aiston ([MA]).

2. Cohomological refinements. The homology of the surgered manifold $M=\mathbf{S}^{3}(L)$ can be described using a Mayer-Vietoris argument. We want to give a precise statement for the group $H^{1}(M ; \mathbf{Z} / n)$. The group $H^{1}\left(\mathbf{S}^{3}-L ; \mathbf{Z} / n\right)$ is canonically isomorphic to $(\mathbf{Z} / n)^{m}$. The inclusion map induces a monomorphism $\phi_{L}: H^{1}(M ; \mathbf{Z} / n) \rightarrow H^{1}\left(\mathbf{S}^{3}-L ; \mathbf{Z} / n\right) \simeq$ $(\mathbf{Z} / n)^{m}$ whose image is the kernel of the linking matrix $B_{L}$, reduced modulo $n$. An elementary Kirby move between $L$ and $L^{\prime}$ gives a diffeomorphism between $\mathbf{S}^{3}\left(L^{\prime}\right)$ and $\mathbf{S}^{3}(L)$ (defined up to isotopy). This diffeomorphism induces the isomorphism

$$
\phi_{L, L^{\prime}}: \operatorname{Ker}\left(B_{L}\right) \simeq H^{1}\left(\mathbf{S}^{3}(L) ; \mathbf{Z} / n\right) \rightarrow H^{1}\left(\mathbf{S}^{3}\left(L^{\prime}\right) ; \mathbf{Z} / n\right) \simeq \operatorname{Ker}\left(B_{L^{\prime}}\right)
$$

The formula for the usual positive Fenn-Rourke move is

$$
\phi_{L, L^{\prime}}\left(c_{1}, \ldots, c_{m-1}, 0\right)=\left(c_{1}, \ldots, c_{m-1}, c_{m}^{\prime}\right) \quad \text { with } \quad c_{m}^{\prime}=-\sum_{i<m} b_{i m}^{\prime} c_{i}
$$

Here $L_{m}$ is a trivial component, with framing one, in a ball; the other components of $L$ slide over this component to obtain the link $L^{\prime}, b_{i m}^{\prime}$ is the corresponding coefficient of the matrix $B_{L^{\prime}}$.

This can be used to construct invariants for pairs $(M, \sigma), \sigma \in H^{1}(M ; \mathbf{Z} / n)$. Suppose that the skein module $\mathcal{S}=\mathcal{S}\left(\mathbf{D}^{2} \times \mathbf{S}^{1}\right)$ is $\mathbf{Z} / n$-graded as an algebra

$$
\mathcal{S}=\bigoplus_{\nu=0}^{n-1} \mathcal{S}_{\nu}
$$

Suppose moreover that this grading is compatible with cabling. By this we mean that for any framed link $L=\left(L_{1}, \ldots, L_{m}\right)$ in $\mathbf{D}^{2} \times \mathbf{S}^{1}$, and for any homogeneous elements $x_{1}, \ldots, x_{m}$ of respective degrees $d_{1}, \ldots, d_{m}$, the skein element $L\left(x_{1}, \ldots, x_{m}\right)$ is homogeneous of degree equal to $\sum \lambda_{i} d_{i}$, where $\lambda_{i}$ is the algebraic intersection of $L_{i}$ with a meridian disc. This implies that the twist $t$ and the $c_{x}$ are graded operators.

Proposition 2.1. If the vectors $\omega_{\nu} \in \mathcal{S}_{\nu}, \nu=0, \ldots, n-1$, satisfy the condition

$$
\forall \nu \forall x_{\nu} \in \mathcal{S}_{\nu}\left\langle H_{1}\left(x_{\nu}, \omega_{-\nu}\right)\right\rangle=\left\langle U_{0}\left(x_{\nu}\right)\right\rangle\left\langle U_{1}\left(\omega_{0}\right)\right\rangle \text { and }\left\langle U_{1}\left(\omega_{0}\right)\right\rangle \text { is invertible }
$$

then, provided $\left(c_{1}, \ldots, c_{m}\right)$ lies in the kernel of $B_{L}$,

$$
\frac{\left\langle L\left(\omega_{c_{1}}, \ldots, \omega_{c_{m}}\right)\right\rangle}{\left\langle U_{1}\left(\omega_{0}\right)\right\rangle^{b_{+}}\left\langle U_{-1}\left(\omega_{0}\right)\right\rangle^{b_{-}}}
$$

is an invariant of the surgered manifold $M=\mathbf{S}^{3}(L)$ equipped with the cohomology class $\sigma=\phi_{L}^{-1}\left(c_{1}, \ldots, c_{m}\right) \in H^{1}(M ; \mathbf{Z} / n)$. 
The condition in the hypothesis above can be reduced to a unique equation if the grading satisfies the condition (WG) below (we will say that the algebra $\mathcal{S}$ is well graded).

(WG) For all $\nu$ there exists $y_{\nu} \in \mathcal{S}_{\nu}$ such that $\mathcal{S}_{\nu}=y_{\nu} \mathcal{S}_{0}$ and $\left\langle U_{0}\left(y_{\nu}\right)\right\rangle$ is invertible.

Lemma 2.2. If the grading of $\mathcal{S}$ satisfies $(W G)$, and $\omega_{0} \in \mathcal{S}_{0}$ satisfies

$$
\forall x_{0} \in \mathcal{S}_{0}\left\langle H_{1}\left(x_{0}, \omega_{0}\right)\right\rangle=\left\langle U_{0}\left(x_{0}\right)\right\rangle\left\langle U_{1}\left(\omega_{0}\right)\right\rangle \text { and }\left\langle U_{1}\left(\omega_{0}\right)\right\rangle \text { is invertible }
$$

then the hypothesis of Proposition 2.1 is satisfied.

Proof. Take $\omega_{\nu}=\left\langle U_{0}\left(y_{\nu}\right)\right\rangle^{-1} y_{\nu} \omega_{0}$.

In the interesting known examples, the cohomological invariant, constructed with a given bracket, appears as a refinement of the one without structure in the following precise sense: the latter decomposes as a sum, over all cohomological classes, of the refined ones. The following theorem gives a sufficient condition for existence of a cohomological invariant satisfying such a decomposition property.

TheOREM 2.3. Suppose the grading of $\mathcal{S}$ satisfies $(W G)$, and $\omega_{0} \in \mathcal{S}_{0}$ is such that

$$
(\mathrm{KC})\left\{\begin{array}{l}
\forall x_{0} \in \mathcal{S}_{0}\left\langle H_{1}\left(x_{0}, \omega_{0}\right)\right\rangle=\left\langle U_{0}\left(x_{0}\right)\right\rangle\left\langle U_{1}\left(\omega_{0}\right)\right\rangle \text { and }\left\langle U_{1}\left(\omega_{0}\right)\right\rangle \text { is invertible } \\
\forall \nu \neq 0 \forall x_{\nu} \in \mathcal{S}_{\nu}\left\langle H_{1}\left(x_{\nu}, \omega_{0}\right)\right\rangle=0
\end{array}\right.
$$

then there exists $\omega_{\nu} \in \mathcal{S}_{\nu}, \nu=0, \ldots, n-1$ such that the formula

$$
\tau(M, \sigma)=\frac{\left\langle L\left(\omega_{c_{1}}, \ldots, \omega_{c_{m}}\right)\right\rangle}{\left\langle U_{1}\left(\omega_{0}\right)\right\rangle^{b_{+}}\left\langle U_{-1}\left(\omega_{0}\right)\right\rangle^{b_{-}}}
$$

is an invariant of the surgered manifold $M=\mathbf{S}^{3}(L)$ equipped with the cohomology class $\sigma=\phi_{L}^{-1}\left(c_{1}, \ldots, c_{n}\right) \in H^{1}(M ; \mathbf{Z} / n)$.

Moreover, if $\omega=\omega_{0}+\ldots+\omega_{n-1}$ then

$$
\tau(M)=\frac{\langle L(\omega, \ldots, \omega)\rangle}{\left\langle U_{1}(\omega)\right\rangle^{b_{+}}\left\langle U_{-1}(\omega)\right\rangle^{b_{-}}}
$$

is an invariant of the surgered manifold $M=\mathbf{S}^{3}(L)$ which satisfies the decomposition property

$$
\forall M \quad \tau(M)=\sum_{\sigma \in H^{1}(M ; \mathbf{Z} / n)} \tau(M, \sigma)
$$

The decomposition formula is a consequence of the lemma below, which can be shown as for lemma 1.2 .

LEMMA 2.4. In the hypothesis of theorem 2.3, $\left\langle L\left(\omega_{c_{1}}, \ldots, \omega_{c_{m}}\right)\right\rangle=0$ if $\left(c_{1}, \ldots, c_{m}\right)$ is not in the kernel of $B_{L} \bmod n$.

EXAMPLE 1. In the Kauffman bracket skein theory, the algebra $\mathcal{K}=\mathcal{K}\left(\mathbf{D}^{2} \times \mathbf{S}^{1}\right)$ is $\mathbf{Z} / 2$-graded. The equation (KC) has a solution only if $A$ is a root of unity whose order is congruent to 8 modulo 16 . The corresponding decomposition theorem has been stated in [Bl1]. Note that, although $\mathcal{K} \simeq k[z]$, the degree does not give a usable $\mathbf{Z} / n$-grading on the skein algebra $\mathcal{K}$, for $n>2$. This is because the Kauffman skein relation in the solid torus is not homogeneous with respect to this degree. 
Example 2. Skein theory $\mathcal{L}$ associated with linking. Using Gauss sum computations, we can show that if $N=2^{2 l+1}$, the invariant $Z_{N}$ of section 1 , example 2 , admits a $\mathbf{Z} / 2^{l}$ cohomological refinement.

ExAmple 3. In $[\mathrm{Mu}]$, H. Murakami gives cohomological refinements of the quantum $S U(n)$-invariants. This refinements as well as the corresponding decomposition formula can be obtained using HOMFLY skein theory.

3. Spin type structures. In $[\mathrm{Mu}], \mathrm{H}$. Murakami states also a decomposition formula in which cohomology classes are replaced by some spin type structures (see remark 2.7 in his paper). He observes that for $n=2$ these are spin structures, and the corresponding refinements were studied in $[\mathrm{KM}]$ and [Bl1]. For $n>2$, he only gives a combinatorial description of the structures, and asks for a topological interpretation. We are going to give a topological definition for these structures. From the combinatorial description in the case of 3-manifolds we will then obtain a version of the results of the previous section for 3-manifolds equipped with these spin type structures. More about these structures will be found in $[\mathrm{Bl}]$.

Suppose $n$ is an even integer. Then there exists, up to homotopy, a unique non trivial map $g: B S O \rightarrow K(\mathbf{Z} / n, 2)$. Define the fibration

$$
\pi_{n}: B \operatorname{Spin}(\mathbf{Z} / n) \rightarrow B S O
$$

to be the pull-back, using $g$, of the path fibration over $K(\mathbf{Z} / n, 2)$. For $n=2$ this construction is well known, and $B \operatorname{Spin}(\mathbf{Z} / 2)=B \operatorname{Spin}$ is a classifying space for the universal covering Spin of the group $S O$. The space $B \operatorname{Spin}(\mathbf{Z} / n)$ is a classifying space for the non trivial central extension of the Lie group $S O$ by $\mathbf{Z} / n$. This extension will be denoted by $\operatorname{Spin}(\mathbf{Z} / n)$ in [Bl2] whence the notation $B \operatorname{Spin}(\mathbf{Z} / n)$. Remark that $\operatorname{Spin}(\mathbf{Z} / n)$ is a sub-group of Spin $^{c}$.

Now we can use the fibration $\pi_{n}$ to define structures (see $[\mathrm{St}]$ ). Let $\gamma_{\operatorname{Spin}(\mathbf{z} / n)}=$ $\pi_{n}^{*}\left(\gamma_{S O}\right)$ be the pull-back of the canonical vector bundle over $B S O$.

Definition. A $\mathbf{Z} / n$ spin type structure (or $\operatorname{Spin}(\mathbf{Z} / n)$-structure, or spin structure with $\bmod n$ coefficients) on a manifold $M$ is an homotopy class of fiber maps from the stable tangent bundle $\tau_{M}$ to $\gamma_{S p i n}(\mathbf{z} / n)$.

If non empty the set of these structures, denoted $\operatorname{Spin}(M ; \mathbf{Z} / n)$, is affinely isomorphic to $H^{1}(M ; \mathbf{Z} / n)$, by obstruction theory. Moreover the obstruction for existence is a class $w_{2}(M ; \mathbf{Z} / n) \in H^{2}(M ; \mathbf{Z} / n)$, which is the image of the Stiefel-Whitney class $w_{2}(M)$ by the homomorphism induced by the inclusion of coefficients $\mathbf{Z} / 2 \hookrightarrow \mathbf{Z} / n$.

The Stiefel-Whitney class $w_{2}(M)$ is zero for every compact oriented 3-manifold, hence $\mathbf{Z} / n$ spin type structures exist on a 3-manifold $M=\mathbf{S}^{3}(L)$. The following theorem gives a combinatorial description of these structures. Recall that $M$ is the boundary of a 4 manifold $W_{L}$ called the trace of the surgery. To each $\sigma \in \operatorname{Spin}(M ; \mathbf{Z} / n)$ is associated a relative obstruction $w_{2}(\sigma ; \mathbf{Z} / n)$ in $H^{2}\left(W_{L}, M ; \mathbf{Z} / n\right)$. The group $H^{2}\left(W_{L}, M ; \mathbf{Z} / n\right)$ is free of rank $m=\sharp L$. Taking the coordinates of the relative obstruction we get a map $\psi_{L}: \operatorname{Spin}(M ; \mathbf{Z} / n) \rightarrow(\mathbf{Z} / n)^{m}$. 
THEOREM 3.1. The map $\psi_{L}$ is injective, and its image is the set of those $\left(c_{1}, \ldots, c_{m}\right)$ which are solutions of the following $(\mathbf{Z} / n$-characteristic equation)

$$
B_{L}\left(\begin{array}{c}
c_{1} \\
\vdots \\
c_{m}
\end{array}\right)=\frac{n}{2}\left(\begin{array}{c}
b_{11} \\
\vdots \\
b_{m m}
\end{array}\right)(\bmod n)
$$

Here the $b_{i i}$ are the diagonal values of the linking matrix $B_{L}$.

Proof. First we compute the absolute obstruction $w_{2}\left(W_{L} ; \mathbf{Z} / n\right)=\xi_{*}\left(w_{2}\left(W_{L}\right)\right)$, where $\xi_{*}$ is induced by the morphism of coefficients $\xi: \mathbf{Z} / 2 \hookrightarrow \mathbf{Z} / n$. If $x$ is an integral 2-cycle and $[x]_{a}$ denotes its homology class modulo an integer $a, w_{2}\left(W_{L}\right) \in H^{2}\left(W_{L} ; \mathbf{Z} / 2\right)$ is determined by the equation

$$
\forall x<w_{2}\left(W_{L}\right),[x]_{2}>=x . x(\bmod 2)
$$

Hence $w_{2}\left(W_{L} ; \mathbf{Z} / n\right) \in H^{2}\left(W_{L} ; \mathbf{Z} / n\right)$ is determined by

$$
\forall x<w_{2}\left(W_{L} ; \mathbf{Z} / n\right),[x]_{n}>=\xi(x . x)=\frac{n}{2} x \cdot x(\bmod n)
$$

Now by functoriality, the relative obstruction lives in the inverse image of the absolute one under the map induced by inclusion $H^{2}\left(W_{L}, M ; \mathbf{Z} / n\right) \rightarrow H^{2}\left(W_{L} ; \mathbf{Z} / n\right)$. Using the affine structure over $H^{1}(M ; \mathbf{Z} / n)$, we obtain an affine bijection between $\operatorname{Spin}(M ; \mathbf{Z} / n)$ and this inverse image. Whence the lemma by writing the equation above using coordinates.

As in section 2 there is a formula for the bijection $\psi_{L, L^{\prime}}$ corresponding to a Kirby move. Using the $\mathbf{Z} / n$-characteristic equation we see that the coefficient for an unknotted component with framing \pm 1 is $n / 2$. For the usual positive Fenn-Rourke move, the formula is

$$
\psi_{L, L^{\prime}}\left(c_{1}, \ldots, c_{m-1}, n / 2\right)=\left(c_{1}, \ldots, c_{m-1}, c_{m}^{\prime}\right) \quad \text { with } \quad c_{m}^{\prime}=n / 2-\sum_{i} b_{i m}^{\prime} c_{i}
$$

Proposition 3.2. If the vectors $\omega_{\nu} \in \mathcal{S}_{\nu}, \nu=0, \ldots, n-1$, satisfy the condition

$\forall \nu \forall x_{\nu} \in \mathcal{S}_{\nu}\left\langle H_{1}\left(x_{\nu}, \omega_{n / 2-\nu}\right)\right\rangle=\left\langle U_{0}\left(x_{\nu}\right)\right\rangle\left\langle U_{1}\left(\omega_{n / 2}\right)\right\rangle$ and $\left\langle U_{1}\left(\omega_{n / 2}\right)\right\rangle$ is invertible then, provided $\left(c_{1}, \ldots, c_{m}\right)$ satisfies the $\mathbf{Z} / n$-characteristic equation,

$$
\frac{\left\langle L\left(\omega_{c_{1}}, \ldots, \omega_{c_{m}}\right)\right\rangle}{\left\langle U_{1}\left(\omega_{n / 2}\right)\right\rangle^{b_{+}}\left\langle U_{-1}\left(\omega_{n / 2}\right)\right\rangle^{b_{-}}}
$$

is an invariant of the surgered manifold $M=\mathbf{S}^{3}(L)$ equipped with the $\mathbf{Z} / n$ spin type structure $\sigma=\psi_{L}^{-1}\left(c_{1}, \ldots, c_{m}\right)$.

TheOREM 3.3. Suppose the grading of $\mathcal{S}$ satisfies $(W G)$, and $\omega_{n / 2} \in \mathcal{S}_{n / 2}$ satisfies

$(\mathrm{KS})\left\{\forall x_{0} \in \mathcal{S}_{0}\left\langle H_{1}\left(x_{0}, \omega_{n / 2}\right)\right\rangle=\left\langle U_{0}\left(x_{0}\right)\right\rangle\left\langle U_{1}\left(\omega_{n / 2}\right)\right\rangle\right.$ and $\left\langle U_{1}\left(\omega_{n / 2}\right)\right\rangle$ is invertible $\left\{\forall \nu \neq 0 \forall x_{\nu} \in \mathcal{S}_{\nu}\left\langle H_{1}\left(x_{\nu}, \omega_{n / 2}\right)\right\rangle=0\right.$

then there exists $\omega_{\nu} \in \mathcal{S}_{\nu}, \nu=0, \ldots, n-1$ such that the formula

$$
\tau(M, \sigma)=\frac{\left\langle L\left(\omega_{c_{1}}, \ldots, \omega_{c_{m}}\right)\right\rangle}{\left\langle U_{1}\left(\omega_{n / 2}\right)\right\rangle^{b_{+}}\left\langle U_{-1}\left(\omega_{n / 2}\right)\right\rangle^{b_{-}}}
$$


is an invariant of the surgered manifold $M=\mathbf{S}^{3}(L)$ equipped with the $\mathbf{Z} / n$ spin type structure $\sigma=\psi_{L}^{-1}\left(c_{1}, \ldots, c_{m}\right)$. Moreover, if $\omega=\omega_{0}+\ldots+\omega_{n-1}$ then

$$
\tau(M)=\frac{\langle L(\omega, \ldots, \omega)\rangle}{\left\langle U_{1}(\omega)\right\rangle^{b_{+}}\left\langle U_{-1}(\omega)\right\rangle^{b_{-}}}
$$

is an invariant of the surgered manifold $M=\mathbf{S}^{3}(L)$ which satisfies the decomposition property

$$
\forall M \quad \tau(M)=\sum_{\sigma \in \operatorname{Spin}(M ; \mathbf{Z} / n)} \tau(M, \sigma)
$$

EXAMPLE 1. The invariant coming from the Kauffman bracket skein theory admits a spin refinement (with a decomposition theorem), if $A$ is a root of unity whose order is congruent to 0 modulo 16 ([Bl1]).

ExAmple 2. If $N=2^{2 l}$, the invariant $Z_{N}$ of the skein theory $\mathcal{L}$ associated with linking admits a $\mathbf{Z} / 2^{l}$ spin refinement.

EXAMPLE 3. For $n$ even the $S U(n)$ specialized HOMFLY theory admits $\mathbf{Z} / n$ spin type refinements $([\mathrm{Mu}])$.

4. Towards TQFTs. A universal method for extending an invariant of closed 3manifolds to a whole Topological Quantum Field Theory is described in [BHMV3] (see [At], [Tu2] or [BHMV3] for axioms of TQFT). Roughly speaking we could say that the problem is to have a pasting formula to compute the invariant out of pieces. If a manifold is cut by a separating surface $\Sigma$, each piece gives a vector in a module associated to the surface, and the invariant is obtained using a hermitian form on this module. Such a formula is clear if one proceeds as follows. Take the free module generated by the manifolds with boundary this surface, and divide by the kernel of the natural pairing defined using the invariant of closed manifolds. This has to be computable; precisely we want each module to have finite rank. The problem is reduced a lot if some natural surgery axioms are satisfied. First one needs an extension of the invariant to closed manifolds with (colored) links. (In our language a colored link is a skein element obtained by cabling some framed link.) Second, in most cases, the required index 2 surgery formula, is obtained only after resolving what is called the framing anomaly; this can be done by using $p_{1}$-structures. In [BHMV3], following the lines above, TQFT's are constructed from the Kauffman bracket. It is shown that the modules associated to surfaces (with $p_{1}$-structure) are free, of finite ranks given by the Verlinde formula.

How does this extend to the refined invariants? In joint work with Gregor Masbaum $([\mathrm{BM}])$ this question is solved for the spin invariants derived from the Kauffman bracket. A finiteness theorem and dimension formulae are given, and it is shown that the multiplicativity axiom holds in a $\mathbf{Z} / 2$-graded sense. The relation between the spin theories and the unspun ones is described by a transfer map. This map identifies the 'unspun' module of a surface $\Sigma$ with the invariant part, under a natural $\widetilde{H}_{0}(\Sigma, \mathbf{Z} / 2)$-action, of the modules obtained in the spin theory (more precisely, the zero-graded part of the spin theory). As already noted, understanding other refined theories is our challenge! 


\section{References}

[At] M. F. Atiyah, Topological quantum field theories, Publ. Math. IHES 68 (1989) 175-186.

[BHMV1] C. Blanchet, N. Habegger, G. Masbaum and P. Vogel, Three-manifold invariants derived from the Kauffman bracket, Topology 31 No 4 (1992), 685-699.

[BHMV2] C. Blanchet, N. Habegger, G. Masbaum and P. Vogel, Remarks on the Threemanifold Invariants $\theta_{p}$, in 'Operator Algebras, Mathematical Physics, and Low Dimensional Topology' (R. Herman, B. Tanbay, Ed.), A.K.Peters Research Notes in Mathematics Vol 5, 39-59 (1993).

[BHMV3] C. Blanchet, N. Habegger, G. Masbaum and P. Vogel, Topological Quantum Field Theories derived from the Kauffman bracket, Topology 34 No 4 (1995), 883927.

[Bl1] C. Blanchet, Invariants on three-manifolds with spin structure, Comm. Math. Helv. 67 (1992), 406-427.

[B12] C. Blanchet, On spin type structures related with quantum invariants, in preparation.

[BM] C. Blanchet and G. Masbaum, Topological Quantum Field Theories for surfaces with spin structure, Duke Math. Journal 82 No 2 (1996), 229-267.

[FR] R. Fenn and C. Rourke, On Kirby calculus of links, Topology 18 (1979) 1-15.

[HP] J. Hoste and J. Przytycki, A survey of skein modules of 3-manifolds, in 'Knots 90', Proceedings of the International Conference on Knot Theory and Related Topics, Osaka (Japan), August 15-19, 1990 (A. Kawauchi Ed.), Walter de Gruyter (1992), 363-379.

[Jo] V. Jones, A polynomial invariant for knots via Von Neumann algebras, Bull. Amer. Math. Soc. 12 (1985) 103-111.

[Ka] L. H. Kauffman, State models and the Jones polynomial, Topology 26 (1987) 395407.

[Ki] R. Kirby, A calculus of framed links in $\mathbf{S}^{3}$, Invent. Math. 45 (1978), 35-56.

[KM] R. Kirby and P. Melvin, The 3-manifold invariants of Witten and ReshetikhinTuraev for sl(2, C), Inv. Math. 105 (1991), 473-545.

[KT] T. Khono and T. Takata, Symmetry of Witten 3-manifold invariants for $\operatorname{sl}(n, \mathbf{C})$, Journal of Knot Theory Ram. Vol. 2 No 2 (1993) 149-169.

[Li1] W. B. R. Lickorish, Three-manifold invariants and the Temperley-Lieb algebra, Math. Ann. 290 (1991), 657-670.

[Li2] W. B. R. Lickorish, Calculations with the Temperley-Lieb algebra, Comm. Math. Helv. 67 (1992), 571-591.

[Li3] W. B. R. Lickorish, The skein method for 3-manifolds invariants, J. Knot. Th. Ram. 2 (1993), 171-194.

[MA] H. R. Morton and A. K. Aiston, Young diagrams, the Homfly skein of the annulus and unitary invariants, Proceedings of Knots 96, Tokyo (to appear).

[Mo] H. R. Morton, Invariants of links and 3-manifolds from skein theory and from quantum groups, In 'Topics in knot theory', the Proceedings of the NATO Summer Institute in Arzurum, NATO ASI Series C 399 (M. Bozhüyük Ed.), Kluwer (1993), 107-156.

[ML] S. Mac Lane, Categories for the working mathematician, Graduate Texts in Math. 5, Springer-Verlag (1971). 
[MOO] H. Murakami, T. Othsuki and M. Okada, Invariants of three-manifolds derived from linking matrices of framed links, Osaka J. Math. 29 (1992), 545-572.

[MPR] J. Mattes, M. Polyak and N. Reshetikhin, On invariants of 3-manifolds derived from abelians groups, in 'Quantum Topology' (L. Kaufmann, R. Baadhio Ed.), World Scientific (1993).

[Mu] H. Murakami, Quantum invariants for 3-manifolds, Proc. of the 3rd Korea-Japan School of knots and links (to appear).

[OY] T. Ohtsuki and S. Yamada, Quantum SU(3) invariants via linear skein theory, J. Knot Theory Ram. 6 (1997), 373-404.

[P] J. Przytycki, Skein modules of 3-manifolds, Bull. Ac. Pol. Math. 39(1-2) (1991), 91-100.

[RT] N. Reshetikhin and V. Turaev, Invariants of 3-manifolds via link polynomials and quantum groups, Invent. Math. 103 (1991), 547-598.

[St] R. E. Stong, Notes on cobordism theory, Princeton Univ. Press (1968).

[Tu1] V. G. Turaev, State sum models in low-dimensional topology, Proc. ICM Kyoto 1990, vol I, 689-698.

[Tu2] V. G. Turaev, Quantum invariants of knots and 3-manifolds, De Gruyter Studies in Math. 18 (1994).

[TW] V. G. Turaev and H. Wenzl Quantum invariants of 3-manifolds associated with classical simple Lie algebras, Int. Journal of Math. Vol. 4 No 2 (1993) 323-358.

[Vo] P. Vogel, Les invariants récents des variétés de dimension 3, Séminaire Bourbaki 799 (1995).

[Wi] E. Witten, Quantum field theory and the Jones polynomial, Comm. Math. Phys. 121 (1989), 351-399.

[Yo] Y. Yokota, Skeins and quantum $S U(N)$ invariants of 3-manifolds, Math. Ann. 307 (1997), 109-138. 